\title{
Spin wave contribution to the specific heat of ferromagnetic terbium at low temperatures
}

\author{
C S MENON \\ Department of Physics, University of Calicut, Calicut University 673635 India. \\ MS received 6 April 1985; revised 30 May 1985
}

\begin{abstract}
The magnon contribution to the specific heat of ferromagnetic terbium is evaluated and the results are compared with the measurements from $90 \mathrm{~K}$ down to absolute zero.
\end{abstract}

Keywords. Spin wave contribution; specific heat; magnon contribution; ferromagnetic terbium.

Recently there has been a spurt in studies on heavy rare earth metals because of the magnon-phonon interactions observed in them. The phonon dispersion relations in terbium have been investigated by Houmann and Nicklow (1970) and by Menon and Rao (1972). The magnon dispersion relations have been measured by Moller et al (1968). In this paper we report the magnetic specific heat of terbium from $90^{\circ} \mathrm{K}$ down to absolute zero.

The total energy $U_{m}(T)$ of the spin waves which are excited at a temperature $T$ is given by

$$
U_{m}(T)=\int_{0}^{\infty} \frac{E g(E) \mathrm{d} E}{\operatorname{Exp}(E / K T)-1}
$$

where $g(E) \mathrm{d} E$ is the number of magnons in the energy interval $E$ and $E+\mathrm{d} E$. The energy density function has been obtained by making use of the measured dispersion relations of Moller et $a l(1968)$ and the normalized energy density function $g(E)$ has been divided into steps of $0.3 \mathrm{meV}$. The integration has been done using the numerical integration method on BESM-6 computer. A direct differentiation of (1) with respect to $T$ will therefore yield the magnetic specific heat $C_{m}(T)$ of the material due to the excitation of the spin waves at that particular temperature. The resultant expression is used to evaluate the magnetic specific heat of terbium in the temperature range 0 to $90^{\circ} \mathrm{K}$.

Figure 1 shows the temperature variation of the magnetic specific heat of terbium. The solid line is the theoretical curve and the dark circles are the experimental values obtained by Brooks et al (1968) from the experimental data of Jennings et al (1957). Upto $20 \mathrm{~K}$, the agreement between the calculated and observed values is excellent. Between $20 \mathrm{~K}$ and $65 \mathrm{~K}$ the experimental values are consistently lower than the calculated ones. Between $75 \mathrm{~K}$ and $90 \mathrm{~K}$ the experimental values are higher than the present calculated values. The maximum discrepancy is found at $30 \mathrm{~K}$ where the deviation is about $28 \%$. The origin of the discrepancy between theoretical and the 


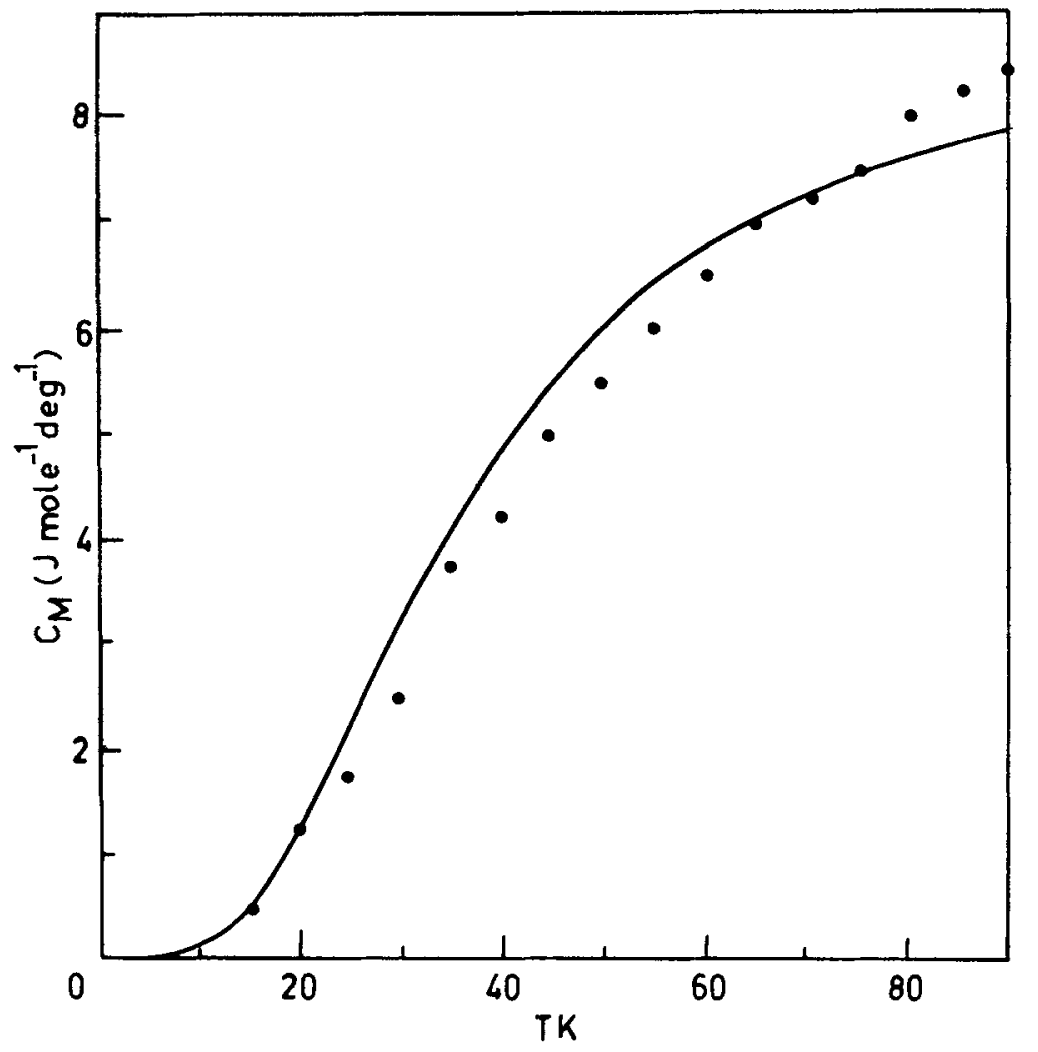

Figure 1.

experimental results above $T=20 \mathrm{~K}$ may be attributed to the assumptions made during the analysis of the experimental data.

It may be mentioned that in the analysis of the specific heat of many of the rare earths, previous workers (Lounasmaa 1966; Lounasmaa and Sundstrom 1966, 1967) had to make fairly drastic assumptions concerning the phonon contribution to the specific heat. As an approximation, they assumed that both the magnetic and the nonmagnetic heavy rare earths have the same lattice specific heat. Hence a thorough reanalysis of the specific heat data of the rare earths is useful.

\section{Acknowledgement}

The author would like to thank Prof. S C K Nair for his encouragement.

\section{References}

Brooks M S, Goodings D A and Ralph H I 1968 J. Phys. C1 1596

Houmann J C G and Nicklow R M 1970 Phys. Rev. B1 3943

Jennings L D, Stanton R M and Spedding F H 1957 J. Chem. Phys. 27909 
Lounasmaa O V 1966 Phys. Rev. 143399

Lounasmaa O V and Sundstrom L J 1966 Phys. Rev. 150399

Lounasmaa O V and Sundstrom L J 1967 Phys. Rev. 158591

Menon C S and Rao R R 1972 Solid State Commun. 10179

Moller H B, Houmann J C G and Mackintosh A R 1968 Appl. Phys. 39807 